\title{
SIGHTPLAN: AN ARTIFICIAL INTELLIGENCE TOOL \\ TO ASSIST CONSTRUCTION MANAGERS WITH SITE LAYOUT
}

\author{
Iris D. Tommelein, Research Assistant \\ Raymond E. Levitt, Associate Professor \\ Department of Civil Engineering \\ and \\ Barbara Hayes-Roth, Senior Research Associate \\ Department of Computer Science \\ Stanford University \\ Stanford, CA 94305
}

\begin{abstract}
SightPlan is an expert system that performs construction site layout. The system contains an explicit representation of the strategy it follows to construct a solution. Based on the assumption that the cognitive capabilities of an agent have an impact on the strategy that agent follows during problem-solving, we decided to experiment with alternate strategies. We modeled one strategy after the way in which a construction field manager would lay out a site (the 'Expert Strategy'), and we compared this model with a second one designed to make better use of the power provided by the computer (the 'Computational Strategy'). The results of this experiment, the comparison of both models, and the implications of this research are described in this paper. They allow us to conclude that AIbased computer programs assisting experienced managers in solving their task may result in better solutions than either the stand-alone machine or the individual person could obtain.
\end{abstract}

\section{INTRODUCTION}

Site layout consists of identifying, sizing, and locating temporary facilities on a construction site. In practice, usually a field manager or a superintendent is assigned to draw up the layout plan at the beginning of construction. This preliminary layout plan describes the site when the project is started, displays the temporary facilities at that time, and shows facilities overlapping with the space allocated for major activities that will take place in the near- and long-term future.

Research work in the 60's and 70's [1] formalized the layout process by identifying optimization criteria and by developing computer programs that could generate the best layout procedurally. When an optimum solution could not be guaranteed, criteria were relaxed and implementations would make use of heuristic methods and improvement procedures. Though these layout models were applied on some construction projects, the approach did not find general acceptance in industry, whereas for instance procedures for optimum location of single facilities $[2 ; 3]$ proved to be more acceptable. We suggest that one of the reasons for their failure is that the black-box procedures for layout were too far removed from the way field practitioners do their job. Because they could not relate to it they would refuse the effort required to collect all needed data. Construction managers interested in disseminating their knowledge on site layout therefore had to resort to writing guidelines [4], checklists [5;6;7], or describing their experience from specific cases [8].

Though the shortcomings of algorithmic models were soon identified (see, e.g., [9] for early ideas on the need for man-machine interactive systems in construction management) it took several years for AI programming techniques to develop, mature [10; 
11], and finally be introduced in construction [12]. A proof of concept that an expert system could model site layout was delivered by $[13 ; 14 ; 15]$. Our interest in applying AI to site layout was (1) to understand and model closely how field practitioners lay out a site, (2) to investigate if we could improve upon their method, and if so, how we would do that; and (3) in case we could not do better, we would try to rationalize where our approach failed. To investigate this, we limited the scope of our expert system model, named SightPlan, to two-dimensional spatial layout, we focused on one specific project site, and we implemented two different layout strategies for comparison.

\section{A CASE STUDY FOR CONSTRUCTION SITE LAYOUT}

SightPlan tackles the layout of the temporary facilities on the Intermountain Power Project (IPP), a coal-fired power plant of two 750MW units located in Delta, Utah (Figure 1). Coal-fired power plant construction is well-established as far as construction practice goes, we had field manuals with guidelines for this type of site layout, and we obtained excellent cooperation from managers of each of the parties involved, so we could learn about their experience on the project.

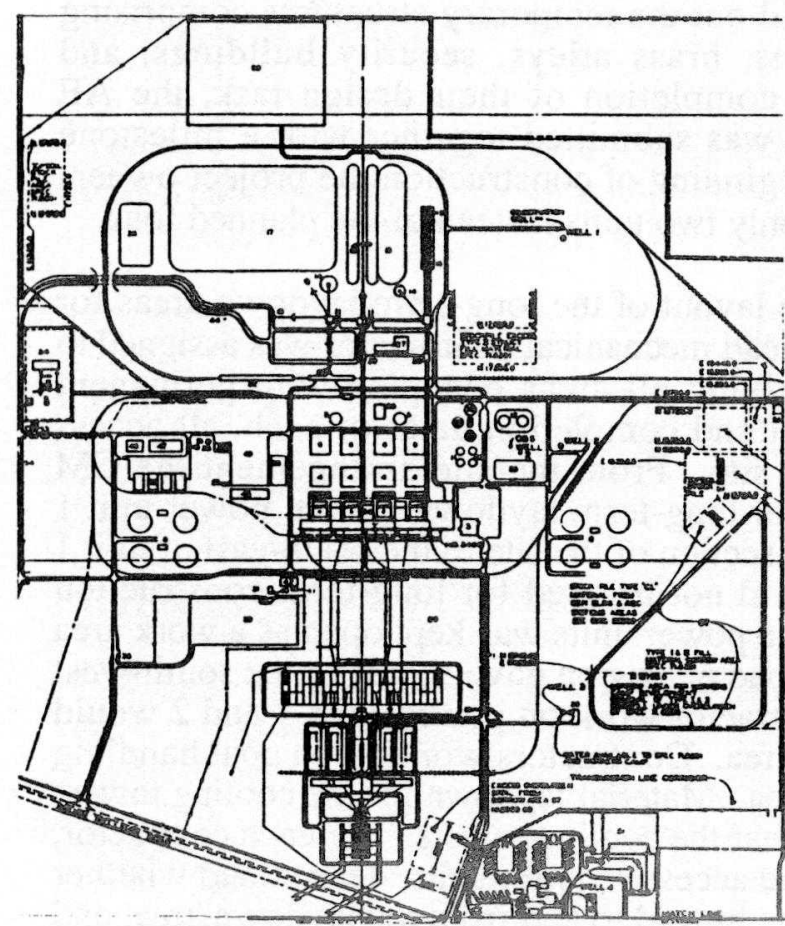

Figure 1: Site Arrangement Drawing of the Intermountain Power Project

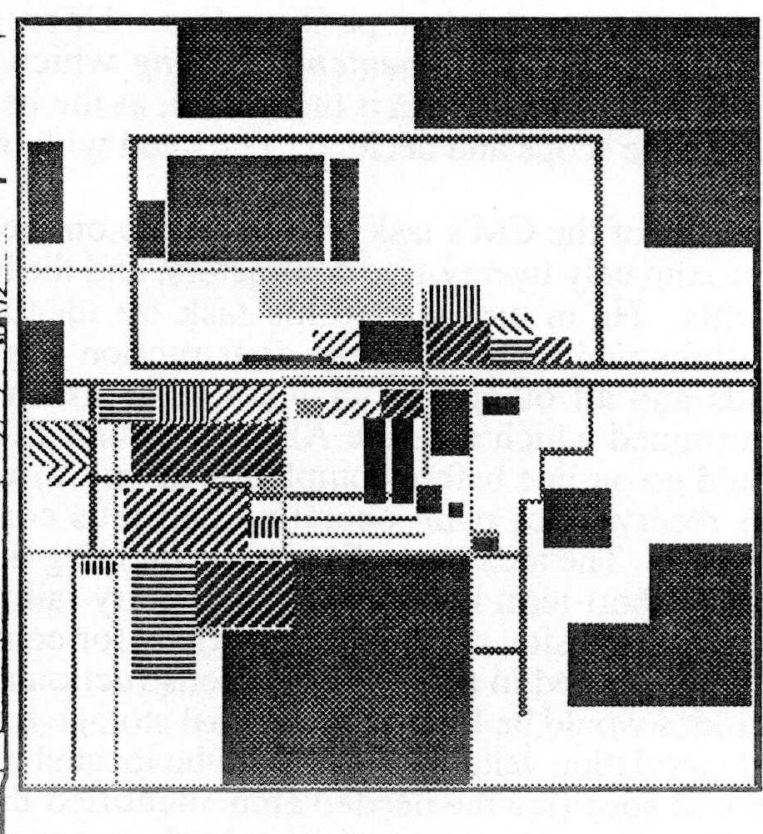

Figure 2: Layout Generated by SightPlan using its 'Expert Strategy'

The program is implemented in BB1 which is a domain-independent blackboard architecture $[16 ; 17]$; a detailed description of the implementation is provided in [18]. Suffice it to say here that the system's knowledge base includes the permanent facilities, each of the facilities to be located, their area, and the dimensions of the rectangular space they would occupy, as well as the geometrical constraints to be satisfied between them in a solution layout. In addition to this, the system has two sets of so-called knowledge sources. The domain knowledge sources describe the actions to take for constructive assembly of the layout. This method consists of deciding stepwise on starting a partial arrangement, including certain facilities, picking a facility that needs to be positioned, satisfying constraints between it and other facilities, or merging arrangements at appropriate 
times. The control knowledge sources describe the strategy SightPlan should follow in selecting which action to take next. So they determine which intermediate steps towards the solution to take and in what order to take them, which ultimately impacts the solution itself. The latter set can thus capture how a person goes about laying out a site, and this is what is described by the term 'Expert Strategy'. We then tried to improve upon this strategy and tailored the 'Computational Strategy'.

\section{EXPERT STRATEGY}

The first model emulates the strategic decisions and steps taken by an experienced field manager while laying out the site. It is therefore called the 'Expert Strategy'. We learned different parts of our layout strategy from the owners, the architect-engineers (AE) and the construction managers (CM) who worked on IPP. The process they described was then simplified so that we could model it. For example, we ignored the vertical dimension of spatial facilities, we did not model the layout of underground utilities, nor did we reason about the short-term laydown areas.

Besides designing the permanent facilities, including power units, support buildings, permanent roads and railroads, the $\mathrm{AE}$ also laid out the temporary structures comprising warehouses, office spaces, first aid facilities, brass alleys, security buildings, and management and labor parking lots. Upon completion of their design task, the AE produced a site arrangement drawing which was submitted together with a milestone schedule to the CM. As it turned out, at the beginning of construction the project owners revised the scope and decided to proceed with only two units instead of the planned four.

Part of the CM's task was to decide on the layout of the long-term laydown areas for approximately twenty-five contractors, and the lead mechanical coordinator was assigned to do this. He in turn started his task by identifying all areas occupied with permanent facilities while checking the construction start and completion dates of each, all access roads and all otherwise unavailable areas on site. From the site arrangement the CM determined which area the AE had provided for long-term laydown. Since power unit 1 would go on-line before completion of unit 2, a section of the site to the south-east of unit 1 was reserved for plant operation and thus could not be used for long-term construction laydown. The area immediately surrounding the power units was kept open as a work area and for short-term laydown. A temporary railroad extension gave access to the south-west corner of the site, so all laydown areas for contractor work on power units 1 and 2 would be concentrated in that so-called construction area. Contractors working on coal handling facilities would be located in the coal storage area. Material laydown for the cooling towers and circulation water piping would be located near the cooling towers. For each contractor, the CM specified the needed area, identified the access requirements, determined whether or not major pieces of material would need to be moved to and from the laydown area, and established how critical the contractor's activity was. Based on this information he ranked the areas by overall importance and picked the first one to find an appropriate location for it on site. This meant: figuring out what area that laydown had to be in (zoning constraint), whether or not it needed to be adjacent to a railroad (adjacency constraint), and of course it could not overlap with roads or any of the fixed facilities on site (non-overlap constraint). Finally, if several alternative positions would remain after meeting these constraints, the preference constraint of the contractor-that is, to be as close as possible to the place of installation of the work in the permanent facility-would be met by picking the best in-thatsense position from the alternatives. Then, the CM would repeat this process with the second contractor's laydown, and so on. In short, the CM used an early commitment strategy in finding positions for contractor laydown areas on site.

The SightPlan model of this Expert Strategy resulted in the layout shown in Figure 2. 
The second model follows a Computational Strategy that we crafted so that it could exploit the power available in our computer model. We could improve upon the Expert Strategy in two ways: (1) by reaching the same results in a shorter computation time, and (2) by generating alternative solutions that were excluded by the early commitment strategy because the latter applied preference constraints before all facilities had met their zoning, adjacency, and non-overlap constraints.

Leaving aside the system's computation for deciding on which action to take next, the main factor to study in order to improve the run-time of the Expert Strategy was the constraint engine. The constraint engine is that module of code that takes as input sets of possible positions of facilities plus constraints, and reduces those to output only positions where the facilities satisfy the imposed constraint. Its efficiency depends on the complexity of the input, and on the types of function calls that are needed to compute constraint satisfaction. As is discussed in [19], it may turn out that applying one constraint before the other on certain facilities can be more efficient than applying the constraints in reverse order. The second factor to improve the Expert Strategy stemmed from the observationand well-known fact-that early commitment may not prove capable of producing a solution, in cases where postponed or least commitment might. We removed the preference constraints from the model. As a result of that of course, the system was unable to pick particular instances from sets of alternative positions. This is what we term a least commitment strategy: all constraints that need to be met are applied to compute the set of feasible positions of facilities, which are dependent upon currently feasible positions of other facilities, yet no further commitment is made to picking a single instance. To replace the preference constraints, the strategy got modified to sample several instances from each set. This is what we term a postponed commitment strategy. It is less restrictive than the early commitment but more restrictive than the least commitment strategy. Subsequently SightPlan would generate coherent instances for the layout, that is, find combinations of facilities - each at one of its sampled positions - at which they do not overlap with one another. In that way, the Computational Strategy could generate many solution layouts for this particular project. Finally, in order to pick one coherent instance of the layout to be the solution, the choice could be based on some evaluation function taking into account user preferences or other criteria.
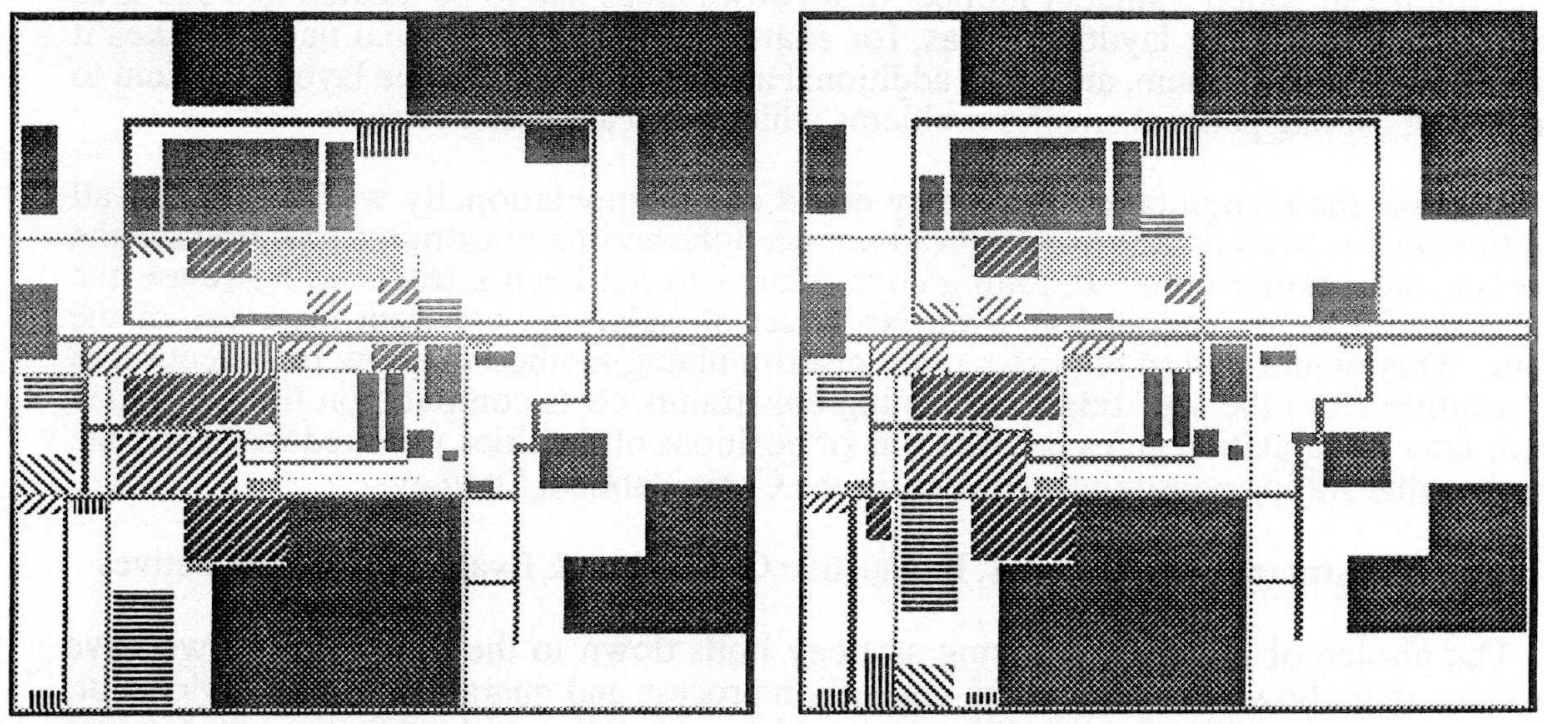

Figure 3: Two Alternative Layouts Generated by SightPlan's 'Computational Strategy' 
This Computational Strategy was applied to the input provided by the AE on their site arrangement drawing; so it is an alternative to the Expert Strategy discussed above. Two of the alternative layouts it generated are shown in Figure 3.

\section{COMPARISON}

In the way we have implemented this work, the Computational Strategy and especially the Expert Strategy are caricatures of respectively a brute-force computer-based approach and a person's strategy. But the experiment was worthwhile because the comparison we can now make provides data to demonstrate the strength and weakness of computer models that try to implement the actions taken by human experts while performing their task, and vice versa, it emphasizes some of the cognitive capabilities as well as limitations of people.

\subsection{Heuristics for Early Commitment vs. Postponed Commitment}

As IPP's project site was very spacious, the layout problem faced by SightPlan turned out to be highly underconstrained. In this instance, the early commitment strategy used by the CM actually worked efficiently: a solution was reached in a small number of steps and it was difficult to improve on this in the Computational Strategy. In general though, early commitment cannot guarantee that it will work at all. Yet, the reason for which people often resort to it is out of necessity, due to their cognitive limitations (see, e.g., [19]): it is difficult to keep many facilities, constraints, and sets of positions in mind at all times, so one alternative is to only focus on one or a few facilities and position them one at a time.

Since computers have plenty of memory, the Computational Strategy can postpone commitment or pursue least commitment: all sets of all possible positions of facilities can be stored easily - albeit at some cost-and the strategy can switch its attention back and forth between facilities without 'getting confused'.

\subsection{Partial Arrangements vs. Global Arrangements}

People actually introduce constraints specifically to make a problem like site layout more tractable. The CM introduced zoning constraints to reduce the problem to several sub-problems in which a smaller number of laydown areas had to be located in a sub-area of the site. Classifying laydown areas, for example by their functional needs, makes it easier to keep track of them, and as an additional advantage it reduces the layout problem to several, almost independent smaller problems which often are easier to solve.

Because the Computational Strategy could deal computationally with laying out all facilities on the overall site at once, it did not need the zoning constraints to subdivide the problem into smaller ones. If zoning constraints had not been introduced however, the system would have generated an even larger set of satisfying coherent instances. of the layout. This would in turn require a more discriminating method to allow for selection of one solution from the set. Imposing zoning constraints costs computation time, but may permit later computation gains because sets of positions of facilities were reduced early on. Therefore the zoning constraints were left in the Computational Strategy.

\subsection{Restricted Generation vs. Exhaustive Generation \& Evaluation of Alternatives}

The choice of a problem-solving strategy boils down to the question: do we have heuristics that allow us to restrict the generation process and guarantee good solutions, or, how much computation are we willing to spend on generating and evaluating alternatives that might otherwise be excluded? Neither early commitment nor least commitment solve 
the site layout problem in a satisfactory manner. Intelligently postponing commitment may be the best intermediate strategy, but exactly how to do this demands more investigation.

To investigate this we implemented a third model for SightPlan. Here, the system first uses hierarchical aggregation to lay out the temporary facilities that were positioned by the $\mathrm{AE}$ on the site arrangement drawing, then performs the task of the $\mathrm{CM}$ as described above by the Expert Strategy. The de-facto separation of tasks between AE and CM is reflected in this two-step strategy. It would not be difficult to alter the strategy and integrate the two layout steps into one, but whether or not that is desirable raises the issue of efficiency. Current practice is indeed to restrictively generate a layout for the $A E$, then to proceed and restrictively generate the $\mathrm{CM}$ layout. Better solutions could possibly be found by the integrated strategy, if the cost of evaluating alternatives is not too high. The balance of this trade-off currently tilts towards restrictive generation with the rationale that AE's facilities are almost as important as the permanent ones: most of these are not really 'temporary' in that they remain on the site after completion of construction of the project and are then rebuilt to serve as maintenance facilities during plant operation. Therefore, first finding the best position for them totally outweighs the gains obtained by locating temporary facilities in a better location. Providing more general generation and better evaluation functions may shift that imbalance, resulting in integrated design-construct-operate strategies.

\subsection{Language for Spatial Representation and Reasoning}

Finally, we have to address how well any of our SightPlan models could possibly perform as compared to how well a construction manager performs. First, computers don't have the cognitive capabilities that people have, and second, the knowledge transfer between humans and machine is far from flawless. The principal issues here are that (1) it is hard to verbalize spatial thinking (field managers would sometimes be unable to articulate how much space had been used for a certain area, but when given a layout plan, they could easily draw the area on it); (2) it is difficult to communicate to another person what exactly is envisioned; and (3) it is hard to represent imprecise ideas of layouts and shapeless areas in geometrical two-dimensional computer graphics.

[19] provides a detailed description and a more rigorous discussion of the work presented here.

\section{JOINT COGNITIVE SYSTEM}

If we want to understand and model closely how field practitioners lay out a site, then learning about field practice provides a good starting point. AI programming techniques permit us to build systems that capture reasonably well the way people perform their task, so that we can actually model an expert's strategy and people could easily relate to the expert model thus built.

We claim however, and we hope to have demonstrated in this experiment, that the exact duplication of actions that mimic human behavior may not be appropriate or even be desirable. People have their cognitive strengths; computers have their computational strengths. In order to benefit from both we provided for a system in which both could collaborate.

Figure 4 shows the current hardware setup to run SightPlan. SightPlan, the expert system that performs the reasoning about what actions to take to lay out the site, runs on a Texas Instruments Explorer ${ }^{\mathrm{TM}}$. It communicates with SightView, the remote interactive graphics system that displays the layout of the partial arrangements, running on a Macintosh II ${ }^{\mathrm{TM}}$. Users can observe SightPlan perform its preferred actions or they can 
make their own changes to the layout on the interactive display. That information is then sent back to SightPlan which incorporates it in its knowledge base and performs further reasoning about it.

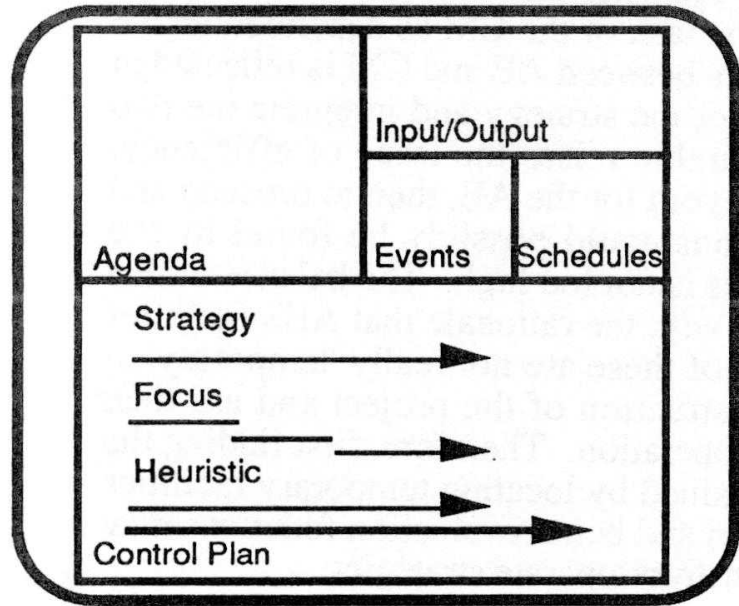

SightPlan

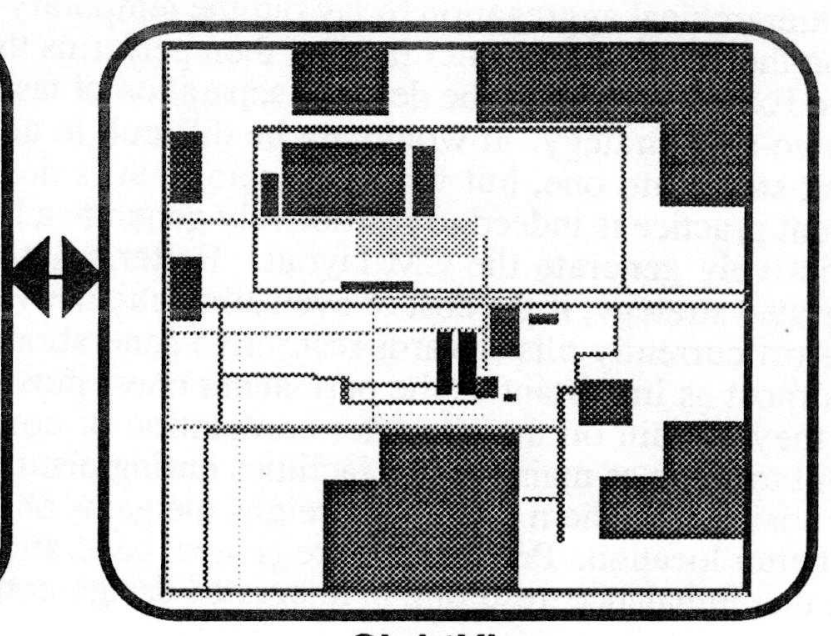

SightView

Figure 4: SightPlan Expert System with Remote SightView Interactive Graphical Display

In this way we built a tool to help people in their task. Human performance is thus augmented in the following ways: (1) SightPlan represents, keeps track of, and reasons about all possible positions at different stages of problem-solving. The user can reduce those sets of positions, but does not need to memorize everything; (2) SightPlan represents multiple partial arrangements and decides which one to work on at the appropriate time; it displays multiple solutions and can evaluate objective functions on them, or allows the user to pick a preferred layout; (3) SightView provides interactive graphics which allow the user to visualize what the current state of the layout is. This setup provides an environment for systematic exploration of alternative layouts.

\section{CONCLUSIONS AND SUGGESTED DIRECTIONS FOR FUTURE RESEARCH}

From this experiment we conclude that some of the system's limitations can be overcome by making it into an interactive tool to be used by construction practitioners, so that the joint cognitive system will perform better in doing site layout than either of its computer or human components would individually.

We find that SightPlan is quite successful in executing its restricted assignment. The system was capable of recreating a layout similar to that produced by the CM from the heuristics in the Expert Strategy, the knowledge sources for constructive assembly, and the set of facilities to be located. We recognize however that the strategy-though stated in general terms - needs to be refined in order to gain flexibility in laying out substantially different or tightly constrained sites, or in acknowledging user modifications to the layouts.

To make SightPlan into an operational support tool for project management, it also needs more knowledge on the identification of space requirements for facilities. If SightPlan had access to multiple project databases it could attempt to learn from previous cases, it could improve on methods to estimate needs and correlate its strategy to site conditions. Furthermore, SightPlan could communicate not only with people via remote displays, but also with other programs, for instance expert systems for planning or scheduling, as space is only one resource to be allocated on construction sites. 


\section{ACKNOWLEDGEMENTS}

Many thanks to all managers at the Los Angeles Department of Water\&Power, Bechtel Construction, Black\&Veatch, and the Intermountain Power Project for their time and patience in providing us with so much information on their work and in granting us permission to reproduce IPP documents. We also acknowledge Tony Confrey's development of the graphical interface and the constraint engine (together with François Daube) for SightPlan, and other people on the BB1 group for their most valuable input. This research was funded by NSF Grant \# MSM-86-13126.

\section{REFERENCES}

This list of references had to be restricted because of space limitations. For a more complete list see [18].

[1] Francis, R.L., White, J.A.: Facility Layout and Location, Prentice-Hall, 468 pages, 1974

[2] Warszawski, A., Peer, S.: Optimizing the Location of Facilities on a Building Site, Operational Research Quarterly, Vol. 24, No. 1, pp.35-44, 1973

[3] Rodriguez-Ramos, W.E., Francis, R.L.: Single Crane Location Optimization, ASCE Journal of Construction Engineering and Management, Vol. 109, No. 4, pp. 387-397, December 1983

[4] Neil, J.M.: Steam-Electric Generating Station Construction, $M-K$ Power Group, section on Construction Site Layout, pp.7-11 to 7-29, June 1982

[5] Neil, J.M.: Teaching Site Layout for Construction, ASCE Meeting, Portland, OR, April 1418, pp.1-11, 1980

[6] Popescu, C.: Temporary Facilities-Utilities Designing Steps, ASCE Convention and Exposition, Portland, OR, 20 pages, 14-18 April 1980

[7] Handa, V., Lang, B.: Construction Site Planning, Construction Canada 88 05, pp. 43-49, 1988 and Construction Site Efficiency, Construction Canada 89 01, pp. 40-48, 1989

[8] Tatum, C.B., Harris, J.A.: Construction Plant Requirements for Nuclear Sites, ASCE Journal of the Construction Division, Vol. 107, No. CO4, December 1981

[9] Paulson, B.C.Jr.: Man-Computer Concepts for Planning and Scheduling, ASCE Journal of the Construction Division, pp. 275-286, September 1972

[10] Waterman, D.A.: A Guide to Expert Systems, Addison-Wesley, 419 pages, 1986

[11] Buchanan, B.G.: Artificial Intelligence as an Experimental Science, Stanford University, Report No. KSL-87-03, 41 pages, January 1987, to appear in Synthese

[12] Levitt, R.E., Kunz, J.C.: Using Knowledge of Construction and Project Management for Automated Schedule Updating, Project Management Journal, Vol. 16, No. 5, Dec. 1985

[13] Hamiani, A.: CONSITE: A Knowledge-Based Expert System for Site Layout, ASCE Proceedings of the 5th Conference, Computing in Civil Engineering: Microcomputers to Supercomputers, Alexandria, Virginia, March 29-31, 1988

[14] Tommelein, I.D., Johnson, Hayes-Roth, B., M.V., Jr. Levitt, R.E.: SIGHTPLAN: A Blackboard Expert System for Construction Site Layout, in J.S. Gero (ed.), Expert Systems in Computer-Aided Design, North Holland, Amsterdam, pp. 153-167, 1987

[15] Tommelein, I.D., Levitt, R.E., Hayes-Roth, B.: Using Expert Systems for the Layout of Temporary Facilities on Construction Sites, in Lansley, P.R., Harlow, P.A. (eds.), Managing Construction Worldwide Volume One: Systems for Managing Construction, Spon, London, pp. 566-577, September 1987

[16] Hayes-Roth, B.: BB1: An Architecture for Blackboard Systems that Control, Explain, and Learn about their own Behavior, Tech. Report HPP-84-16, Stanford University, 1984

[17] Hayes-Roth, B.: A Blackboard Architecture for Control, Artificial Intelligence, Vol. No. 26, pp. 251-321, 1985

[18] Tommelein, I.D.: SightPlan-An Expert System That Models and Augments Human Decision-Making for Designing Construction Site Layouts, $\mathrm{PhD}$ Dissertation, Department of Civil Engineering, Stanford University, Stanford, CA, June 89 (forthcoming)

[19] Tommelein, I.D.: Comparing Design Strategies of Agents with Limited Resources, MSAI Thesis, Dept. of Computer Science, Stanford University, Stanford, CA, June 89 (forthcoming)

[20] Miller, G.A.: The Magical Number Seven, Plus or Minus Two: Some Limits on our Capacity for Processing Information, Psych.Review, Vol. 63, No. 2, pp. 81-97, March 1956 